\title{
Variations
}

Variations

Revue internationale de théorie critique

$9 / 10 \mid 2007$

Les frontières de la politique

\section{Ce que le mot prolétariat signifie aujourd'hui}

\section{Oskar Negt et Alexander Kluge}

Traducteur : Alexander Neumann

\section{(2) OpenEdition}

\section{Journals}

Édition électronique

URL : http://journals.openedition.org/variations/465

DOI : $10.4000 /$ variations. 465

ISSN : 1968-3960

Éditeur

Les amis de Variations

Édition imprimée

Date de publication : 1 juin 2007

Pagination : 28-36

Référence électronique

Oskar Negt et Alexander Kluge, «Ce que le mot prolétariat signifie aujourd'hui », Variations [En ligne],

9/10 | 2007, mis en ligne le 01 décembre 2012, consulté le 03 mai 2019. URL : http://

journals.openedition.org/variations/465 ; DOI : 10.4000/variations.465

Ce document a été généré automatiquement le 3 mai 2019.

Les ami•e•s de Variations 


\title{
Ce que le mot prolétariat signifie aujourd'hui
}

\author{
Oskar Negt et Alexander Kluge
}

Traduction : Alexander Neumann

1 Kluge : Prenons le terme " prolétarien ». Que faut-il entendre par là?

2 Negt : L'adjectif "prolétarien» désigne un caractère, lequel renvoie au substantif prolétariat. Je pense qu'il devient difficile d'utiliser ce concept substantiel, car celui-ci suggère une étendue sémantique qui n'existe plus dans les faits.

3 Kluge : Le prolétariat industriel a existé dans certains pays comme la Grande-Bretagne, tandis qu'en Chine il a été limité à la ville de Shanghai ; est-ce correct?

4 Negt : Bien évidemment, il y a la classe ouvrière industrielle. Le concept de prolétariat qui lui correspond est issu du XIXe siècle. À travers ce terme, Marx saisit la possibilité de transformer historiquement les conditions d'existence de la classe ouvrière. Lorsque Marx parle du prolétariat, il pense à la fonction d'une chose, à savoir les ouvriers de l'industrie. Dans le même temps, il considère que le prolétariat constitue un levier, susceptible de changer sa propre condition existentielle, grâce à une démarche organisée. Pourtant, le mot prolétariat provient de l'époque de la Rome antique, où le terme décrit une situation complètement statique.

5 Kluge: Les proles, ce sont les jeunes gens des faubourgs ou des banlieues, qui ne participent pas aux décisions politiques...

6 Negt : Aux yeux de l'Etat romain, ceux-là ne représentent qu'une pure perte, des gens qui ne font que boire et manger.

$7 \quad$ Kluge : Ils ne font même pas des soldats.

8 Negt : Pas même des soldats; ils n'ont pratiquement aucun droit. Cette idée se trouve ensuite transposée vers le XIXe siècle, où elle se trouve promue au rang d'un substantif.

9 Kluge : Selon le procédé classique qui veut que l'on affirme une conscience de soi, en transformant une appellation méprisante en un concept positif. 
10 Negt : Oui, cela rappelle la disposition des sièges à l'Assemblée, selon le clivage gauche/ droite. Au départ, on a assigné les sièges du côté gauche aux rebelles, à ceux qui contestent l'ordre ; cette assignation finit par produire la conscience de soi de la gauche.

Kluge : En partant d'une insulte.

Negt : L'insulte devient alors valeur positive.

Kluge : Cette signification ne peut plus être effacée ensuite.

Negt : Non, elle ne part plus. L'aspect prolétarien fait toujours appel à des expériences particulières des dominés; il s'agit donc d'une caractérisation des expériences dominées. Cela veut dire que le concept prolétarien absorbe des processus sociaux concrets, qui concernent à la fois l'oppression et son possible dépassement.

Kluge : Si l'on veut aujourd'hui retrouver la piste de ce concept, il faut sans doute l'aborder à partir d'une série de questions : où est passé ce mot, qui n'est jamais évoqué par la presse? Où sont passés les phénomènes qui correspondaient à ce terme? En transformant ainsi des affirmations en questionnements, n'obtiens-je pas un concept de recherche pertinent?

Negt : L'une de ces questions serait de savoir s'il convient de saisir le prolétariat dans sa dimension sensible, à travers la solidarité collective, c'est-à-dire à partir d'une expérience qui s'enracine dans une situation vécue, alors que son contraire se caractérise par l'abstraction, se présentant comme une chose séparée, globalisée, dépourvue de cette expérience.

7 Kluge : De cette façon, il est possible de développer des variations à partir du substantif «prolétariat». La question opposée serait alors de savoir s'il existe des situations où l'expérience de la domination, la privation des moyens de production, provoque la recherche d'une issue, qui fait que les hommes produisent de nouveaux instruments? Autrement dit, la domination peut-elle mener à l'autonomie?

Negt : C'est la question décisive.

Kluge : Ou est-ce que la domination ne produit que des dépressions?

Negt : Non, ce n'est pas le cas, mais elle peut sans doute créer un cercle dépressif, qui capte les hommes au fur et à mesure qu'on leur enlève leurs propres moyens de production visibles, les réduisant à une attitude purement réactive. La réflexion sur cette expropriation représente en soi déjà un nouveau moyen de production.

21 Kluge : La domination rend aussi malade, la domination peut également s'avérer explosive. N'a-t-on pas vu qu'elle pouvait déboucher sur des mobilisations de masse, par exemple dans le fascisme?

Negt : Oui.

Kluge : Cette orientation explosive, non productive, destructrice, conquérante et agressive, ne représente-t-elle pas un autre caractère prolétarien?

Negt : C'est pourquoi l'aspect prolétarien ne se limite pas au groupe ouvrier, mais peut être retrouvé dans d'autres milieux sociaux, chez d'autres personnes, sous la forme de processus prolétariens, dont l'un des versants est l'expropriation. À chaque fois se pose la question des racines : comment se forme la vie, comment se produit-elle, quelles sont les conditions d'existence, du développement et du changement de la vie ? Ce raisonnement reste lié à l'aspect prolétarien, à mon sens, en dépit du fait que l'association d'idées dominantes provoquée par le mot prolétariat au sein de l'espace public, n'en tient pas 
compte, notamment l'idée de la misère, de la paupérisation absolue. Bien évidemment, il est possible de désigner ce prolétariat, dans sa forme traditionnelle, par exemple dans les quartiers de misère du tiers-monde. Cependant, aucun être humain, doté d'une identité propre et d'une faculté de juger, ne sort d'une machinerie abstraite... suppose l'existence d'une identité humaine, identité qui ne peut pas être créée à travers la flexibilité elle-même. La fragmentation des activités humaines conduit à une perte d'identité. Les gens ne peuvent pas sans cesse changer de rôle. Si certains en sont néanmoins capables, sans tomber malades, alors ils s'appuient sur des processus antérieurs de formation de l'identité. Ces processus impliquent l'existence de relations personnalisées, de relations de confiance ainsi que toute une série d'autres aspects qui dépendent de relations de proximité.

Kluge : Le système de la création de la vie in vitro s'arrête théoriquement au moment de l'accouchement. À partir de la naissance, jusqu'à la première maturité, chaque enfant doit nécessairement être élevé par des hommes et des femmes. Eux seuls sont en mesure de transmettre l'expérience de la vie grâce à un lien de proximité, qui est toujours d'ordre humain et jamais assuré par une machine. 

incorporer la morale de travail qui fait que l'on ne les contraint plus physiquement à se présenter sur leurs lieux de travail. Cette puissante restructuration sociale se fonde sur une expropriation initiale, dans un double sens : d'une part, il y a expropriation au sens littéral, en ce qui concerne la propriété, mais d'autre part, il s'agit de la dépossession de certaines facultés, par exemple celles des artisans, qui sont intégrés dans la machinerie industrielle. Cela signifie que l'accumulation primitive s'empare de tout l'être humain et non seulement de ses biens. Depuis toujours, différentes formes de résistance et de contestation ont accompagné ce mode d'expropriation.

Kluge : Schumpeter prétend que la destruction créative des conditions existentielles des hommes leur permet de trouver de nouvelles idées. Selon cette lecture, l'homme est au fond un être paresseux et conservateur, qui a besoin d'être poussé en avant par la violence objective des rapports extérieurs. L'école de Chicago et ses représentants du néolibéralisme considèrent également que pareilles atrocités constituent une loi 
d'évolution, depuis l'évolution biologique du genre humain. Je pense qu'il faut déplacer l'angle de vue. Marx dirait sans doute : je vais observer et exposer les choses, un peu comme le font Schumpeter et les néolibéraux, mais je dis que tout cela ne peut pas se faire en dehors des relations humaines de proximité, de solidarité et en dehors de la quête du bonheur.

Negt : Je suis tout à fait d'accord. Marx a bien saisi le fait que le capitalisme profite gratuitement de la capacité des êtres humains à travailler. Cette condition est créée par les femmes, qui assurent et maintiennent la capacité à travailler des futurs adultes. Les lecteurs qui ont eux-mêmes des enfants, ou ceux qui fréquentent des enfants, savent que le processus qui précède la capacité de travail est extrêmement exigeant. Ils savent aussi que cet effort n'est pratiquement pas rémunéré.

Kluge : Le terme "prolétarien" ne désigne pas seulement une certaine temporalité sociale et un mode de production social particulier, mais touche aussi au rapport entre le travail et la nature.

50 Negt : Je pense que cette façon de saisir le concept permet de lui donner un sens approprié à la situation actuelle. Le côté prolétarien rappelle ainsi un aspect de la vie paysanne, à savoir le soin que l'on porte aux outils de production et à la nature.

Kluge : À la manière dont on travaille un champ?

52 Negt: Oui, à l'origine, même le terme «culture » est employé de façon similaire, notamment par Cicéron dans les «Dialogues de Tusculum». Il y parle de la «cultura animi ", c'est-à-dire de la manière dont on laboure...

Kluge :... l'esprit.

Negt :... les sens, les âmes et les esprits. Il comprend la culture comme un processus de production. À mon sens, cette dimension prolétarienne peut être transposée vers le mode de travail des intellectuels. Les activités mentales nécessitent des facultés artisanales, afin que leur productivité et leur créativité puissent se déployer, donc elles intègrent aussi un élément agraire. On peut dire que nous portons tous en nous la trace de l'artisan ou du paysan, une survivance de leur expérience de la souffrance, de la domination et du dépassement de cette domination...

Kluge : Et du retour au pays.

Negt : Une trace de l'exode et du retour, ou de l'expropriation et de la réparation à un niveau supérieur. Car aucune expulsion ne crée d'effets productifs si elle se solde uniquement par le retour à la situation initiale; l'expropriation n'est pas productive si elle aboutit à une restauration. Le retour qui intègre le changement signifie un rétablissement des facultés originaires qui comprend un élément productif et réflexif. $\mathrm{Vu}$ ainsi, l'aspect prolétarien décrit une dimension constitutive de notre vie: la réappropriation de ce qui a été exproprié.

Kluge : J'aimerais relater une petite histoire que Heiner Müller a souvent évoquée. Le Sud de la Russie pendant la Seconde Guerre mondiale. Nous sommes donc à un moment où des armées ouvrières industrielles luttent l'une contre l'autre. Un soir, un prisonnier soviétique, conducteur de char, est assis non loin d'un char de l'armée allemande. Les Allemands sont assis un peu plus loin. L'espace d'un instant, le regard du conducteur russe s'arrête sur la chenille du char allemand. L'un des conducteurs de char allemands remarque et suit ce regard; il voit qu'une vis est en train de se défaire. Le lendemain, la chenille pourrait tomber si la vis n'était pas fixée. Le regard du Russe exprime un certain 
regret. Il y a un élément prolétarien dans ce type d'entendement qui se déroule en dehors de l'hostilité militaire. préoccupe d'un défaut de production. Cet aspect a toujours appartenu au concept de prolétariat: il ne s'agit pas d'une solidarité de façade, mais d'êtres humains qui produisent ensemble leurs conditions d'existence. Ils produisent leur propre vie et dépendent les uns des autres; ils dépendent de la reconnaissance d'autrui au sein de ces processus de production. Par conséquent, ils se comportent comme des êtres humains, qui ne cherchent pas des boucs émissaires de leur misère, qui ne se fondent pas sur des sentiments d'exclusion, tout en vivant le processus de production comme un destin commun. Le travail en commun leur confère leur identité et leur donne leur force.

61 Kluge : C'est une partie intégrante de tous les modes de production connus, sans que nous arrivions à désigner clairement cette partie, en tant que partie séparée.

Negt : Bien souvent, cet aspect est tellement enfoui qu'il devient difficile de le discerner.

Kluge : Cette partie prolétarienne ne construit pas de grands systèmes.

Negt : Beaucoup de gens pensent qu'une personne connectée sur Internet devient une personne créative et productive. Il le devient plutôt s'il en a déjà eu la capacité avant de se connecter, donc s'il investit sa créativité initiale dans la toile...

Kluge : Sa faculté s'accroît encore lorsqu'il rencontre d'autres qui partagent ses qualités.

Negt : J'appelle cela l'extension des organes, qui devient possible grâce à l'utilisation de la technique. 